\title{
DNA sequences alignment in multi-GPUs: acceleration and energy payoff
}

\author{
Jesús Pérez-Serrano ${ }^{1}$, Edans Sandes² ${ }^{2}$ Alba Cristina Magalhaes Alves de Melo² and Manuel Ujaldón ${ }^{*}$ \\ From 5th International Work-Conference on Bioinformatics and Biomedical Engineering \\ Granada, Spain. 26-28 April 2017
}

\begin{abstract}
Background: We present a performance per watt analysis of CUDAlign 4.0, a parallel strategy to obtain the optimal pairwise alignment of huge DNA sequences in multi-GPU platforms using the exact Smith-Waterman method.

Results: Our study includes acceleration factors, performance, scalability, power efficiency and energy costs. We also quantify the influence of the contents of the compared sequences, identify potential scenarios for energy savings on speculative executions, and calculate performance and energy usage differences among distinct GPU generations and models. For a sequence alignment on chromosome-wide scale (around 2 Petacells), we are able to reduce execution times from $9.5 \mathrm{~h}$ on a Kepler GPU to just $2.5 \mathrm{~h}$ on a Pascal counterpart, with energy costs cut by $60 \%$.

Conclusions: We find GPUs to be an order of magnitude ahead in performance per watt compared to Xeon Phis. Finally, versus typical low-power devices like FPGAs, GPUs keep similar GFLOPS/w ratios in 2017 on a five times faster execution.
\end{abstract}

Keywords: GPGPU, CUDA, DNA sequences alignment, HPC, Power efficiency

\section{Background}

The rapid evolution of sequencing techniques has produced myriads of data in recent Genome Projects. In this context, data is generated in such a high rate that the traditional analyses tools are not able to cope with them, leading to a dilemma usually called data deluge. Consequently, the focus of Genome Projects has moved from data production to data analysis and the central challenge nowadays is how to analyze such a huge amount of data in a rapid and accurate way. In order to face this challenge, biology and computer science joined forces, exploring solutions that demand sophisticated algorithms and powerful computing devices. As a result, refined solutions have been devised to support several biological subdomains such as protein structure prediction and docking $[1,2]$, sequence comparison [3] and evolutionary biology [4], among others.

\footnotetext{
*Correspondence: ujaldon@uma.es

${ }^{1}$ Computer Architecture Department, University of Malaga, Malaga, Spain Full list of author information is available at the end of the article
}

High performance computing platforms are composed of several computing cores and can accelerate several algorithms from many research domains, producing results in reduced time. Modern GPUs (graphics processing units) have thousands of cores and have proved to be excellent platforms to run parallel applications that exhibit regular data dependencies. They have been with us for a decade as typical accelerators, and have recently found alternatives in Xeon Phi processors as many-core platforms for HPC and FPGAs as low-power devices. CUDA (Compute Unified Device Architecture) [5] and OpenCL [6] provide valuable support for data and compute intensive applications to exploit the GPU's powerful engine, making it possible to attain several TFLOPS (Trillions Floating Point Operations per Second) and high speed bandwidth. GPUs have been used as successful platforms for large-scale bioinformatics and many researchers have investigated the behaviour of these applications on GPUs and suggested improvements [7-9].

This work extends the study to energy consumption, an issue of growing interest in the High Performance

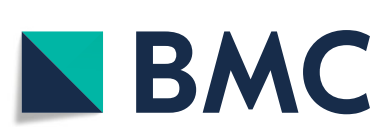

(c) The Author(s). 2018 Open Access This article is distributed under the terms of the Creative Commons Attribution 4.0 International License (http://creativecommons.org/licenses/by/4.0/), which permits unrestricted use, distribution, and reproduction in any medium, provided you give appropriate credit to the original author(s) and the source, provide a link to the Creative Commons license, and indicate if changes were made. The Creative Commons Public Domain Dedication waiver (http://creativecommons.org/publicdomain/zero/1.0/) applies to the data made available in this article, unless otherwise stated. 
Computing (HPC) community. GPU-based supercomputers have been for several years in the top500 list (www. top500.org) of the most powerful supercomputers and have recently conquered the green500 (www.green500. org) supercomputer list. In fact, energy consumption sometimes represents more than $20 \%$ of the budget in Data Centers, and costs have exceeded 5 billion dollars per year over the last decade only in the US [10]. Many estimations state that energy costs will greatly increase in the near future unless power optimizations are applied in all system levels, from the operating system to the application.

This paper is an extended version of [11]. Our work focuses on pairwise biological sequence alignment, aiming to obtain the degree of similarity between the sequences. Within this topic, there are two basic approaches: (a) global alignment, which aligns the entire length of the sequences and is used for similar sequences in content and size; and (b) local alignment, where high similarity sections within the sequences are highlighted. Needleman-Wunsch (NW) [12] proposed an algorithm for global sequence alignment based on dynamic programming (DP), and Smith-Waterman (SW) [13] adapted the NW algorithm to the local alignment case. Both algorithms have high computational requirements, so researchers either use heuristic methods as in the well-known BLAST tools [14], or rely on high performance computing solutions to reduce the execution time. We have chosen GPUs to explore the latter.

The rest of this paper is organized as follows. "Biological sequence comparison" section describes the problem of comparing two DNA sequences. "Related work" section completes this section with some related work. "CUDAlign implementation on GPUs" section summarizes our previous work on GPUs. "Experimental setup" and "Monitoring energy" sections introduce our infrastructure for measuring the experimental numbers, which are later analyzed in "Results" section. Finally, "Discussion and conclusions" section draws conclusions of this work.

\section{Methods}

\section{Biological sequence comparison}

Biological sequences are composed of an ordered sequence of residues, which can be nucleotides (DNA/RNA sequences) or amino acids (protein sequences) [15]. These sequences are treated as strings composed of elements of the alphabets $\Sigma=\{A, T, G, C\}, \Sigma=\{A, U, G, C\}$ and $\Sigma=$ $\{A, C, D, E, F, G, H, I, K, L, M, N, P, Q, R, S, T, V, W, Y\}$, respectively. Protein and RNA sequences are rather small and their sizes range from hundreds to tens of thousands of residues (amino acids and nucleotide bases, respectively). On the other hand, DNA sequences can be very long, often composed of Millions of Base Pairs (MBP).

\section{Similarity score and alignment}

To compare two sequences, we need to find the best alignment between them, that is, how characters match when you overlap them [16]. In an alignment, spaces (gaps) can be inserted in arbitrary locations along the sequences so that they end up with the same size.

In order to measure the quality of a DNA sequence alignment a score is calculated, considering three cases: (a) matches $(m a)$, if the characters of both sequences at the same column are identical; (b) mismatches ( $m i)$, if the characters in the same column are distinct and (c) gaps (gap), if one of the characters in the same column is a space. The score is the sum of all values assigned to the columns and a high score points to high similarity sequences. Figure $1 \mathrm{a}$ and $\mathrm{b}$ illustrate global and local alignments between two DNA sequences $\left(S_{0}=\right.$ ACTTGTCCG and $S_{1}=$ ATGTCAG).

In Fig. 1, a single value is assigned for matches and mismatches $(+1$ and -1 in the example) regardless of the parts involved. This works well with nucleotides (DNA or RNA sequences) but not for proteins. During evolution, some combinations are more likely to occur than others, so higher scores are assigned to these combinations [17]. Therefore, the alignment of protein sequences employ scoring matrices, such as PAM (Percent Accepted Mutations) and BLOSUM (Blocks Substitution Matrix), which associate values for matches/mismatches that correspond to the likelihood of a particular combination [15]. PAM matrices have scores that are calculated by analyzing the frequencies in which a given amino acid is substituted by another amino acid during evolution. BLOSUM scoring matrices are created by evaluating evolutionary rates of a

\begin{tabular}{|ccccccccc}
$A$ & $C$ & $T$ & $T$ & $G$ & $T$ & $C$ & $C$ & $G$ \\
$A$ & - & - & $T$ & $G$ & $T$ & $C$ & $A$ & $G$ \\
\hline+1 & -2 & -2 & +1 & +1 & +1 & +1 & -1 & +1 \\
\hline \multicolumn{10}{c}{ score $=1$}
\end{tabular}

(a)

\begin{tabular}{|c|c|c|c|c|c|}
\hline$T$ & $G$ & $T$ & $C$ & $C$ & $G$ \\
\hline$T$ & $G$ & $T$ & $C$ & $A$ & $G$ \\
\hline+1 & +1 & +1 & +1 & -1 & +1 \\
\hline
\end{tabular}

(b)

Fig. 1 (a) Global and (b) local alignments and scores. Values for matches, mismatches and gaps are $+1,-1$ and -2 , respectively 
region of inside a protein (block) rather than considering the entire protein.

\section{Exact algorithms: obtaining the optimal alignment Algorithm NW for global alignment}

The algorithm proposed by Needleman and Wunsh (NW) [12] is an exact method based on dynamic programming to obtain the optimal global alignment between two sequences in quadratic time and space. In fact, the algorithm originally proposed by NW had cubic time complexity [16], but later on, its complexity was reduced to quadratic, which is the version we describe in this paper. The algorithm is divided in two phases: create the DP matrix and obtain the optimal global alignment.

Phase 1 - calculate the DP matrix In the first phase, the input sequences are $S_{0}$ and $S_{1}$, with $\left|S_{0}\right|=m$ and $\left|S_{1}\right|=n$, where $\left|S_{i}\right|$ is the length of sequence $S_{i}$. For sequences $S_{0}$ and $S_{1}$, there are $m+1$ and $n+1$ possible prefixes, respectively, including the empty sequence. In order to represent the $n$-th character of a sequence $S_{i}$, the notation is $S_{i}[n]$. Fianlly, we use $S_{i}[1 . . n]$ to characterize a prefix with $n$ characters, from the beginning of the sequence.

In the initialization step, the first row and column are set to $-G x$, where $x$ is the size of the non-empty subsequence and $G$ is the gap penalty. This represents the cost of aligning a non-empty subsequence with an empty one. In other words, the first row and column of the DP matrix are initialized with values $H_{0, j}=-G j$ and $H_{i, 0}=-G i$. Additionally, $H_{0,0}=0$. The remaining elements of $H$ are calculated with the recurrence relation Eq. 1 . The optimal score is the value contained in cell $H_{m, n}$.

$$
H_{i, j}=\max \left\{\begin{array}{l}
H_{i-1, j-1}+p(i, j) \\
H_{i, j-1}-G \\
H_{i-1, j}-G
\end{array}\right.
$$

In Eq. 1 , if DNA or RNA sequences are compared, $p(i, j)$ is usually the match value $(m a)$ if $S_{0}[i]=S_{1}[j]$ or the mismatch penalty $(-m i)$, otherwise. If protein sequences are compared, $p(i, j)$ is given by a scoring matrix (e.g. PAM or BLOSUM).

Figure 2 presents the DP matrix between sequences $S_{0}=$ ATTGTCAGGAGG and $S_{1}=$ ACTTGTCCGAGA . The arrows indicate the cell from where the value was obtained, according to Eq. 1. Cells with multiple arrows indicate that the same maximum value was produced by more than one cell $\left(H_{i-1, j-1}, H_{i, j-1}, H_{i-1, j}\right)$.

Phase 2 - obtain the optimal global alignment Phase 2 starts from the position that contains the optimal score $\left(H_{m, n}\right)$ and follows the arrows until cell $H_{0,0}$ is reached. A left arrow in $H_{i, j}$ (Fig. 2) produces the alignment of $S_{0}[i]$ with a gap in $S_{1}$. An up arrow aligns $S_{0}[j]$ with a gap in

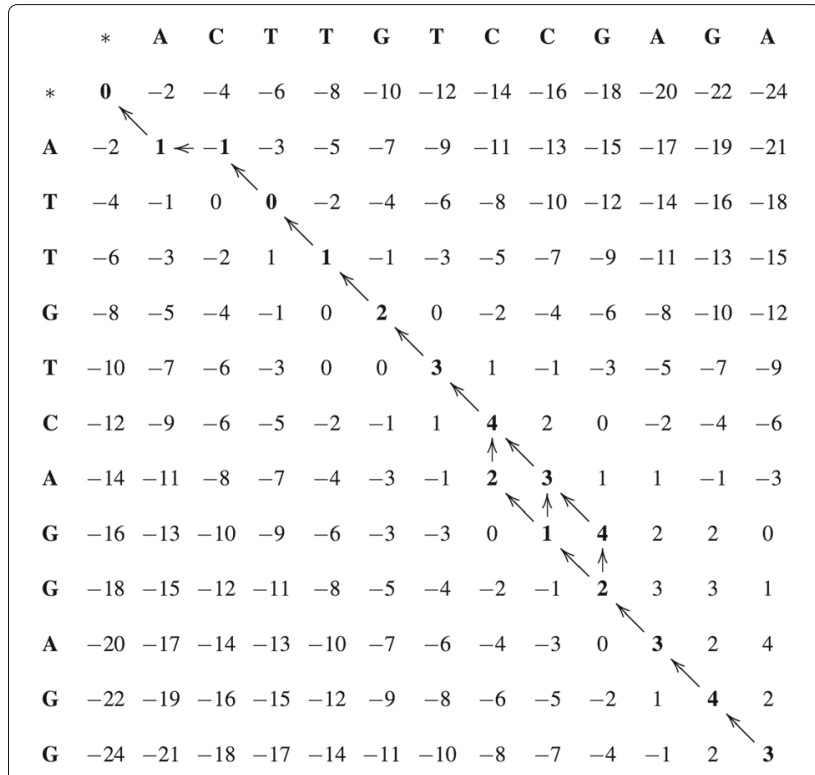

Fig. 2 DP matrix for global alignment between sequences $S_{0}$ and $S_{1}$

$S_{1}$. Finally, a diagonal arrow indicates that $S_{0}[i]$ is aligned with $S_{1}[j]$.

Note that many optimal global alignments may exist, since many arrows may be present in the same cell $H_{i, j}$. Usually, the implementations restrict to one the optimal alignment, giving preference to a given type of arrow (diagonal, up, left).

\section{Algorithm SW for local alignment}

Local alignment must be employed when the goal is to obtain the similarity between regions inside the sequences. Smith and Waterman (SW) [13] proposed an exact algorithm for local alignment 1985 and, since then, this algorithm is widely used. Like NW ("Algorithm NW for global alignment" section), SW is also based on dynamic programming with quadratic time and space complexity. However, there are three basic differences between the algorithms NW and SW, concerning the calculation of the DP matrix ("Phase 1 - calculate the DP matrix" section) and the alignment retrieval.

In the initialization step, all elements of the first row and column are set to zero in SW. This is done because gaps should not receive any penalty at the beginning of the alignment.

The second difference is the recurrence relation itself since since negative values are not allowed in SW, as shown in Eq. 2.

$$
H_{i, j}=\max \left\{\begin{array}{l}
H_{i-1, j-1}+p(i, j) \\
H_{i, j-1}-G \\
H_{i-1, j}-G \\
0
\end{array}\right.
$$


In the second phase (obtain the optimal local alignment), the algorithm starts from the cell which has the highest value in the DP matrix, following the arrows until a zero-valued cell is reached.

Figure 3 presents the similarity matrix to obtain local alignments between sequences $S_{0}=$ TATAGGTAGCTA and $S_{1}=$ GAGCTATGAGGT. Note that, in this example, even though there are no multiple arrows leaving a single cell, two optimal alignments can be obtained, both of them with score 5. Most of the implementations of SW will only retrieve one of those optimal alignments.

\section{Affine-gap}

Algorithms NW ("Algorithm NW for global alignment" section) and SW ("Algorithm SW for local alignment" section) use the linear gap model, where each gap is assigned the same penalty. To produce more biologically relevant results, Gotoh [18] proposed an algorithm that implements the affine-gap model for the global alignment case. This model takes into account the observation that, in nature, gaps tend to occur together [17]. In this case, a higher penalty is assigned to open a gap $\left(G_{\text {open }}\right)$ than to extending it $\left(G_{\text {extend }}\right)$. The cost of a sequence of gaps of length $x$ in the affine gap model is $\gamma(x)=G_{\text {open }}+(x-1) *$ $G_{\text {extend }}$.

The Gotoh algorithm calculates three DP matrices: $H, E$ and $F$, where $H$ keeps track of matches/mismatches and $E$ and $F$ keep track of gaps in each sequence. Time and space complexities are quadratic. Matrix $H$ is calculated with Eq. 3 and matrices $E$ and $F$ use Eqs. 4 and 5, respectively [18]. The optimal score is the value of cell $H_{m+1, n+1}$.

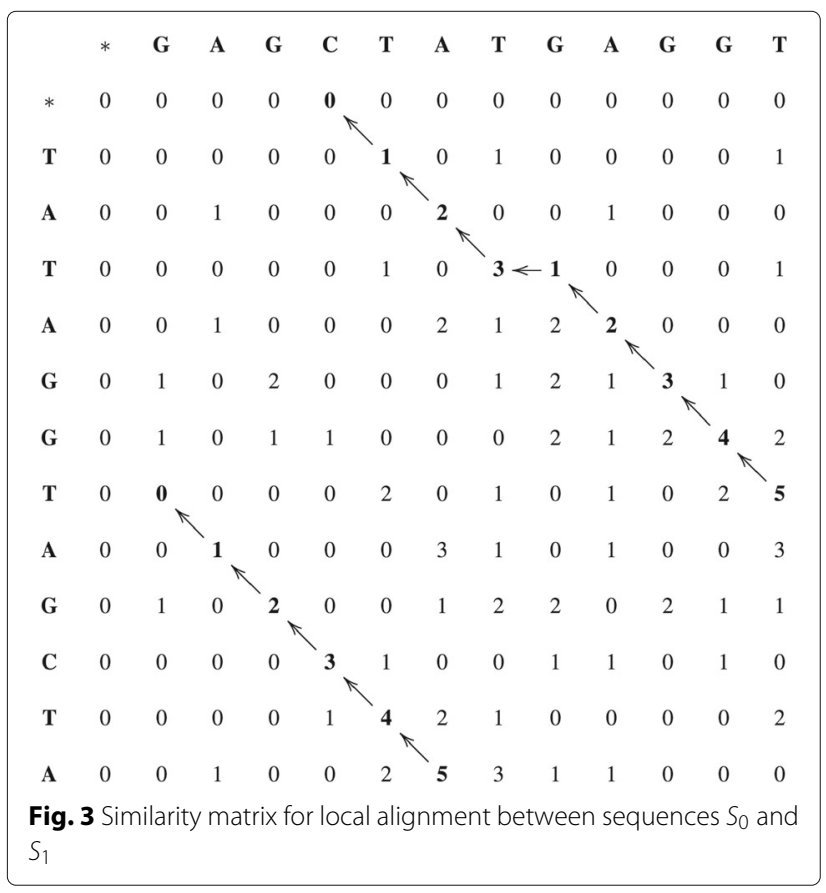

To do the traceback, the arrows are followed in matrix $H$ when a match/mismatch occurs or in matrices $E$ and $F$, to track multiple gaps [18].

$$
\begin{gathered}
H_{i, j}=\max \left\{\begin{array}{l}
0 \\
E_{i, j} \\
F_{i, j} \\
H_{i-1, j-1}-p(i, j)
\end{array}\right. \\
E_{i, j}=\max \left\{\begin{array}{l}
E_{i, j-1}-G_{\text {ext }} \\
H_{i, j-1}-G_{\text {first }}
\end{array}\right. \\
F_{i, j}=\max \left\{\begin{array}{l}
F_{i-1, j}-G_{\text {ext }} \\
H_{i-1, j}-G_{\text {first }}
\end{array}\right.
\end{gathered}
$$

\section{Linear space}

The quadratic space complexity of NW, SW and Gotoh imposes severe restrictions when long sequences are compared. In such cases, linear space algorithms must be used. Hirschberg [19] proposed one of the first linear space algorithms for exact pairwise global sequence comparison [19]. The algorithm employs a recursive divide and conquer strategy that works as follows. First, the DP matrix is computed in linear space from the beginning to the middle row $(i *)$, storing only the last row calculated. Second, the DP matrix is calculated from the end to the middle row over the reverses of the sequences. The algorithm then uses these two middle rows and obtains the position where the addition of both columns $j$ is maximal. This position is called midpoint and it corresponds to an element that belongs to the optimal alignment [19]. The midpoint divides the matrix into two smaller submatrix, which are processed recursively, until trivial solutions are found.

Myers and Miller [20] (MM) adapted Hirschberg's algorithm to the affine gap model ("Affine-gap" section), using two additional vectors to treat situations where a sequence of gaps occurs. Let $i *=\frac{m}{2}$ be the middle row of the DP matrices, $C C(j)$ be the minimum cost of a conversion of $S_{0}[1 . . i *]$ to $S_{1}[1 . . j], D D(j)$ be the minimum cost of a conversion of $S_{0}[1 . . i *]$ to $S_{1}[1 . . j]$ that ends with a gap, $R R(n-j)$ be the minimum cost of a conversion of $S_{0}[i * . . m]$ to $S_{1}[j . . n]$ and $S S(n-j)$ be the minimum cost of a conversion of $S_{0}[i * . . m]$ to $S_{1}[j . . n]$ that begins with a gap.

To find the midpoint of the alignment, the algorithm realizes a matching procedure against a) vectors $C C$ with $R R$ and b) vectors $D D$ with $S S$. The midpoint is the coordinate $(i *, j *)$, where $j *$ is the position that satisfies the maximum value in Eq. 6.

$$
\max _{j \in[0 . n]}\left\{\operatorname { m a x } \left\{\begin{array}{l}
C C(j)+R R(n-j) \\
D D(j)+S S(n-j)-G_{o p e n}
\end{array}\right.\right.
$$


As in Hirshbergś, after the midpoint is found, the matrix is recursively split into smaller submatrix, until trivial solutions are found.

\section{Heuristic algorithms}

Usually, a given protein sequence is compared against thousands or even millions of sequences that compose genomic databases. Also, two long DNA sequences with more than a million base pairs are often compared.

In these scenarios, the use of exact algorithms such as NW and SW is often prohibitive in terms of execution time. For this reason, faster heuristic methods for local alignment were proposed which do not guarantee that the optimal result will be produced. Usually, these heuristic methods are evaluated using the concepts of sensitivity and sensibility. Sensitivity is the ability to recognize as many significant alignments as possible, including distantly related sequences. Selectivity is the ability to narrow the search in order to discard false positives [17]. Typically, there is a tradeoff between sensitivity and sensibility.

The FASTA (FAST-All) algorithm [21] was proposed in 1988 and it computes local alignments of DNA or protein sequences. It is based on FastP [22], which is a heuristic algorithm to compare a protein sequence to a genomic database composed of several protein sequences.

BLAST (Basic Local Alignment Search Tool) [23] was proposed in 1990 and it is based on FASTA. Nowadays, it is the most widely used heuristic tool for local sequence alignment. Like FASTA, the BLAST algorithm assumes that significant alignments have words of length $w$ in common and it is divided into three well-defined phases: seeding, extension and evaluation.

The original BLAST algorithm searched for local alignments without considering gaps. In 1996 and 1997, two improved gapped versions of the original BLAST, NCBIBLAST2 [24] and WU-BLAST2 [25], were proposed.

\section{Related work}

The Smith-Waterman (SW) algorithm has become very popular over the last decade to calculate the optimal pairwise comparison of (1) two DNA/RNA sequences or (2) a protein sequence (query) to a genomic database which is composed of several sequences.

Both scenarios have been parallelized in the literature $[26,27]$, but fine-grained parallelism applies better to the first scenario due to the amount of data and computation involved, and therefore fits better into many-core platforms. Among them, we find Intel Xeon Phis [28], Nvidia GPUs using CUDA [29], and even multi-GPU using CUDAlign 4.0 [30], which is our departure point to analyze cost, performance and power efficiency along this work.

Studies that deal with energy consumption are becoming relevant in DNA sequence comparison applications, which motivates to provide methodologies to measure energy in this context.

Cheah et al. [31] apply an application specific integrated circuit (ASIC) design flow to decrease the power consumption of an accelerator that compares biological sequences. Reduced clock cycles and dynamic frequency scaling are employed to minimize the energy cost.

Hasan and Zafar [32] present a thorough performance versus power consumption study for bioinformatics sequence alignment using distinct field programmable gate arrays (FPGAs). A linear systolic array was used to implement the SW algorithm in those FPGA platforms.

Zou et al. [33] analyze performance and power for SW on CPU, GPU and FPGA, declaring the FPGA as the overall winner. Nevertheless, the authors do not measure real-time power dynamically, but simplify the measurements with a static value for the whole run. Moreover, they use models from the first and second Nvidia GPU generations (GTX 280 and 470), which are, by far, the most inefficient CUDA families as far as energy consumption is concerned (just 4-6 GFLOPS/W versus 15-17 GFLOPS/W in the third generation and up to $40 \mathrm{GFLOPS/W}$ in the fourth generation).

Benkrid et al. [27] perform a similar analysis on CPU, FPGA and GPU, even including the Cell BE. They report 0.085 GCUPS for the CPU, 1.2 GCUPS for the GPU, 3.84 GCUPS for Cell BE and 19.4 GCUPS for the FPGA. Eight years later, we attain 276.53 GCUPS on a Pascal GPU and 37.67 GCUPS on a Altera Stratix V FPGA as we will show later on "Comparison with other devices and implementations" section, where we compare our work with theirs. Additionally, we measure real power on physical wires at real-time, instead of using static values or even TDPs like contemporary authors do [34].

\section{CUDAlign implementation on GPUs}

SW executes in two phases: (1) compute the DP matrix and (2) traceback. Most of the time is spent in phase 1 and, therefore, this phase is often parallelized. Equation 2 exposes the data dependency among the DP matrix cells, i.e., $H_{i, j}$ depends on three other cells: $H_{i-1, j}, H_{i-1, j-1}$ and $H_{i, j-1}$. The parallelization of this kind of dependency is traditionally made by the wavefront method [35], in which each diagonal of the DP matrix is computed in parallel.

The wavefront method is illustrated in Fig. 4. At the beginning (step 1 ) a single cell is calculated in diagonal $d_{1}$. In step 2 , both cells of diagonal $d_{2}$ are computed in parallel. In steps 3 to 5 , the number of cells that can be calculated in parallel increases until it reaches the maximum parallelism in diagonal $d_{5}$. The maximum parallelism is kept in the computation of diagonals $d_{5}, d_{6}, d_{7}, d_{8}$ and $d_{9}$ (five cells are calculated in parallel). In the computation of diagonals $d_{10}$ to $d_{12}$, the parallelism decreases until a single cell is computed in diagonal $d_{13}$. In the wavefront 


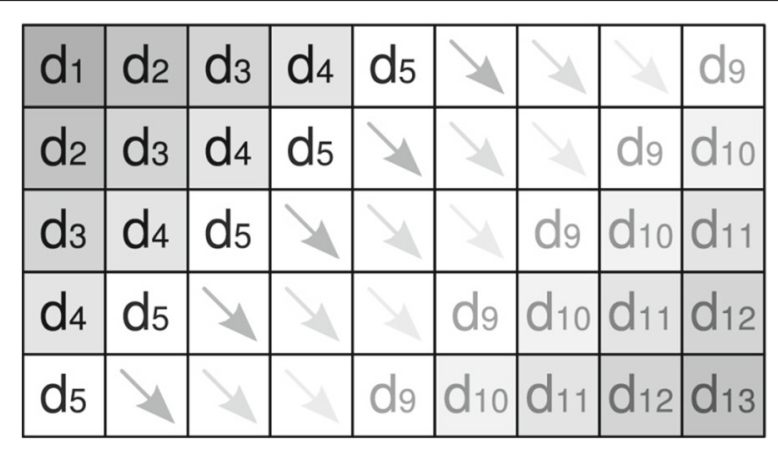

Fig. 4 The wavefront method

method, parallelism is non-uniform and it is explored in a limited way when the wavefront is being filled (beginning of the computation) and when it is being emptied (end of the computation).

CUDAlign [29] obtains the alignment of long sequences with variants of SW and Myers-Miller (see stages and phases summarized in Table 1). The GPU calculates a single SW matrix using all many-cores in a fine grained way, and data dependencies force neighbour cores to communicate in order to exchange border elements.

When CUDAlign is executed in a platform composed of multiple GPUs, a challenging scenario arrises. The computation of the SW matrix is split among the GPUs and each GPU calculates a subset of columns. GPUs are arranged logically in a linear way, sending the border column elements to the next GPU. Communication between neighbor GPUs is overlapped with computations, i.e., it is carried by asynchronous CPU threads while the GPUs keep computing (Fig. 5).

CUDAlign 4.0 [30] introduces two strategies to optimize the traceback phase in multi-GPU executions: Pipeline Traceback (PT) and Incremental Speculative Traceback (IST). PT executes in parallel Stages 2 to 4 from different partitions. First, Stage 1 is executed, and then starts Stage 2 in last GPU. When last GPU gets the crosspoint, this is sent to previous GPU, which starts the execution of Stage 2 for the neighboring partition. The executions of Stages 2, 3 and 4 might be calculated in pipeline at each GPU.

Table 1 Summary of CUDAlign stages, including the SW phase it belongs to and the processor where it is executed

\begin{tabular}{llll}
\hline Stage & Description & SW Phase & Who \\
\hline 1 & Obtaining the optimal score. & 1 & GPU \\
2 & Partial traceback. & 2 & GPU \\
3 & Splitting partitions. & 2 & GPU \\
4 & Balanced splitting. & 2 & CPU \\
5 & Concatenating the optimal alignment. & 2 & CPU \\
6 & Alignment visualization (optional). & 2 & CPU \\
\hline
\end{tabular}

The IST strategy for stage 2 was built based on PT strategy. IST uses the knowledge obtained in several analyses, particularly that the best score in border columns tend to coincide with the crosspoints in them. With this in mind, IST speculates crosspoints in intermediate border columns and Stage 2 in Fig. 5 can be executed in parallel before the optimal alignment crosspoints are known.

\section{CUDAlign versions}

CUDAlign was implemented in CUDA, $\mathrm{C}++$ and pthreads. Results obtained in a large GPU cluster using long DNA sequences present good scalability for up to 16 GPUs [30]. Using CUDAlign 4.0 and input data set described in "Experimental setup" section, a 14.8x speedup was attained. In this case, the execution time was reduced from $33 \mathrm{~h}$ and $20 \mathrm{~min}$ (single GPU) to $2 \mathrm{~h}$ and 13 min (16 GPUs).

Table 2 presents the set of improvements and optimizations performed on CUDAlign over the years. Along this work, we use CUDAlign 4.0.

\section{Experimental setup}

We have conducted an experimental study on a computer endowed with an Intel Xeon server, where we have plugged different number and models of Nvidia GPUs (they will be changing depending of the experiment performed). See Table 3 for a summary of hardware features.

As input, we used real DNA sequences obtained from the National Center for Biotechnology (NCBI) [36]. We compare homologous chromosomes from human and chimpanzee genomes, as it has been observed high similarity in evolutionary studies on the human species [37], in particular for chromosomes 16 [38], 22 [39] and Y [40]. Our selection is summarized in Table 4, where comparisons are named chr22, chr21, 47M, chrY, following names found in $[29,30]$. DNA sequences from all those chromosomes are compared in the results presented in Table 5. From Table 6 on, we always use as input the pair of sequences from the chromosome 22 comparison 


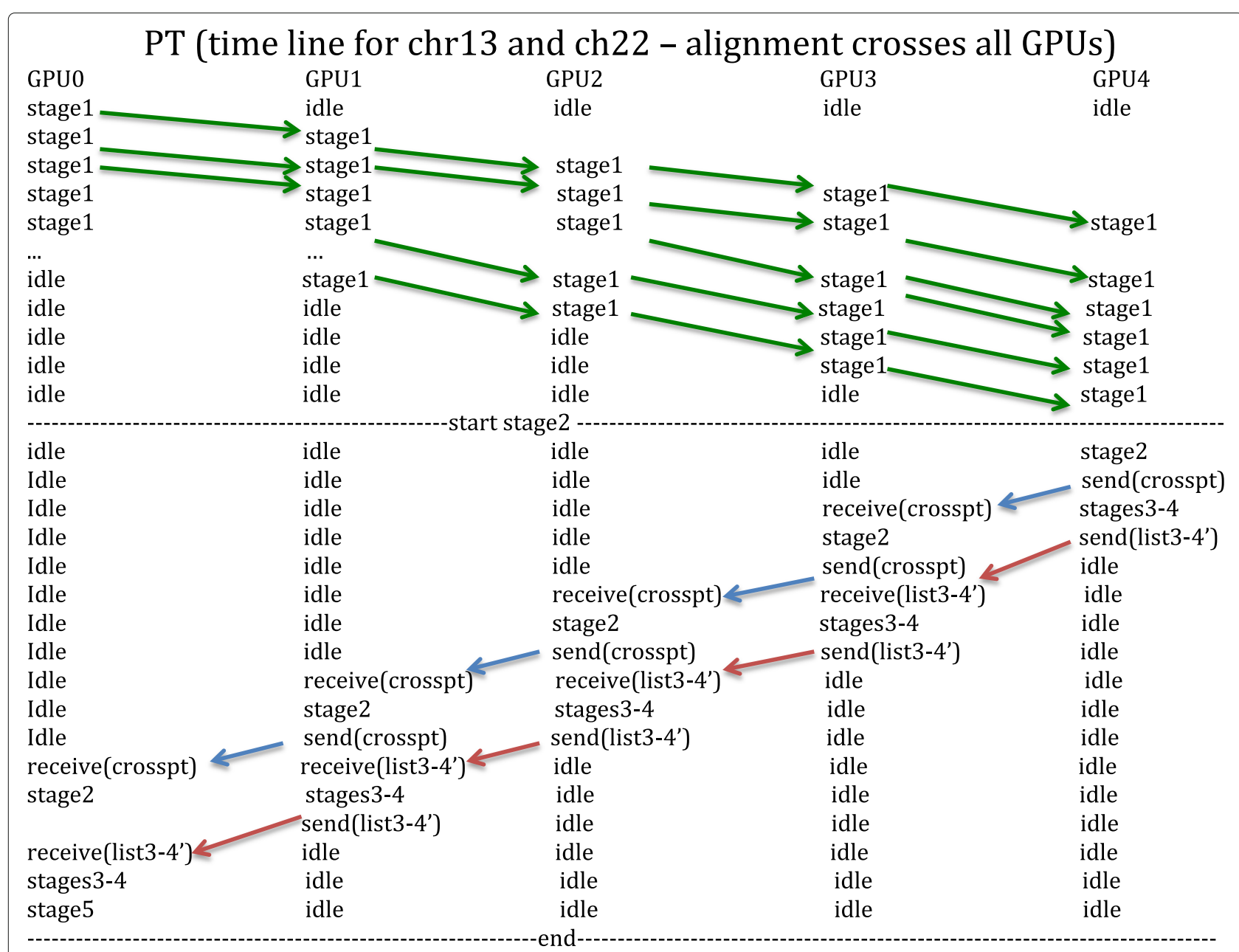

Fig. 5 Timeline for a multi-GPU sequence alignment (4 GPUs)

Table 2 Summary of CUDAlign versions

\begin{tabular}{|c|c|c|}
\hline Version & Major contributions & Ref. \\
\hline 1.0 & $\begin{array}{l}\text { Uses SW with affine gap (Gotoh) to compare long } \\
\text { sequences on GPUs. It ouputs the optimal score and } \\
\text { the end coordinates of the optimal alignment. }\end{array}$ & {$[50]$} \\
\hline 2.0 & $\begin{array}{l}\text { Includes an adapted version of Myers-Miller to retrieve } \\
\text { the optimal alignment in linear space. }\end{array}$ & [51] \\
\hline 2.1 & Block pruning optimization. & [29] \\
\hline 3.0 & $\begin{array}{l}\text { Multi-GPU version for SW phase } 1 \text { (distributes the } \\
\text { matrix, overlaps computations with communications). }\end{array}$ & [49] \\
\hline 4.0 & $\begin{array}{l}\text { Multi-GPU version for SW phases } 1 \text { and 2, including } \\
\text { Incremental Speculative Traceback (IST) to accelerate } \\
\text { the multi-GPU retrieval of the optimal alignment. }\end{array}$ & [30] \\
\hline MASA & $\begin{array}{l}\text { Multi-platform architecture for sequence aligner, } \\
\text { enabling versions to run on (1) multicore CPU using } \\
\text { OpenMP, (2) multicore CPU using OmpsSs, (3) many- } \\
\text { core GPU using CUDA, and (4) Xeon Phi using } \\
\text { OpenMP. }\end{array}$ & [52] \\
\hline
\end{tabular}

(chr22) between the human (51.30 MBP [41], accession number NC_000022.11) and the chimpanzee (49.73 MBP [42], accession number NC_006489.4).

The execution of the required stages on a multi-GPU platform required two modifications in CUDAlign 4.0: (1)

Table 3 Characterization of the infrastructure (CPU and GPUs)

\begin{tabular}{|c|c|c|c|c|c|}
\hline Processor & Intel Xeon CPU & Nvidia G & eForce GP & & \\
\hline Model & $\mathrm{E} 5-2620 \mathrm{v} 4$ & GTX 680 & GTX 980 & $\operatorname{Titan} \mathrm{X}$ & Titan X \\
\hline Generation & Broadwell-EP & Kepler & Maxwell & Maxwell & Pascal \\
\hline Year & 2017 & 2013 & 2015 & 2016 & 2017 \\
\hline Number of cores & 8 & 1536 & 2048 & 3072 & 3584 \\
\hline Core speed (MHz) & 2100 & 1006 & 1216 & 1000 & 1405 \\
\hline GFLOPS (peak) & 16,8 & 3090 & 4980 & 7144 & 10157 \\
\hline Memory Size (GB) & 64 & 2 & 4 & 12 & 12 \\
\hline "Speed (MHz) & 2400 & 6000 & 7000 & 7000 & 10000 \\
\hline "Width (bits) & 256 & 256 & 256 & 384 & 384 \\
\hline " Bandwidth (GB/s) & 76,8 & 192 & 224 & 336 & 480 \\
\hline
\end{tabular}


Table 4 The input data set used along our experiments

\begin{tabular}{lllll}
\hline $\begin{array}{l}\text { DNA } \\
\text { sequence } \\
\text { comparison }\end{array}$ & chr22 & Chr21 & $47 \mathrm{M}$ & ChrY \\
\hline $\begin{array}{l}\text { Human size } \\
\text { Human }\end{array}$ & N1.304.566 & 48.129 .895 & 46.944 .323 & 59.373 .566 \\
$\begin{array}{l}\text { accession \# } \\
\text { Chimpanzee }\end{array}$ & 49.737 .984 & 46.489 .110 & 32.799 .110 & 26.342 .871 \\
size & & & & \\
Chimpanzee & NC_006489.4 & NC_006488.2 & BA0000046.3 & NC_006492.3 \\
accession \# & & & & \\
Petacells & 2.55 & 2.24 & 1.54 & 1.56 \\
Score & 31.510 .791 & 36.006 .054 & 27.206 .434 & 1.394 .673 \\
Length & 51.929 .087 & 48.579 .349 & 33.583 .457 & 2.283 .191 \\
Coverage & $98.9 \%$ & $99.0 \%$ & $70.5 \%$ & $6.0 \%$ \\
Matches & $88.5 \%$ & $91.9 \%$ & $94.4 \%$ & $88.1 \%$ \\
Mismatches & $3.8 \%$ & $1.1 \%$ & $1.5 \%$ & $2.0 \%$ \\
Gaps & $7.7 \%$ & $7.1 \%$ & $4.1 \%$ & $10.0 \%$ \\
\hline
\end{tabular}

partitioning the input sequences to fit them in texture memory, and (2) saving extra rows to the file system as marks to be used in later stages to find crosspoints with the optimal alignment. Furthermore, our experimental analysis is done on the first three stages of CUDAlign, which are the ones executed on GPUs as shown in Table 1. Stages 4 and 5 contribute with marginal execution times, and porting codes to the GPU would not be amortized, whereas stage 6 represents the external visualization.

\section{Monitoring energy}

Our system to measure current, voltage and wattage was built based on a Beaglebone Black, an open-source hardware [43] combined with the Accelpower module [44], which has eight INA219 sensors [45]. As described in [46], we consider two power pins on the PCI-express slot (12 and 3.3 volts) plus six external 12 volts pins coming from the power supply unit in the form of two supplementary 6-pin connectors (see Fig. 6).

The Accelpower module uses a modified version of pmlib library [47], a software package specifically created for monitoring energy. A server daemon collects power data from devices and sends them to the clients, together with a client library for communication and synchronization with the server.

Our procedure for measuring energy begins with a startup of the server daemon. Then, the CUDAlign 4.0 source code was modified in order to include the measurement calls within the GPU code as shown in Fig. 7. Before launching the code, we have to (1) declare pmlib variables, (2) clear and set the wires which are plugged to the server, (3) create a counter and (4) start it. At the end of the GPU execution, we (5) stop the counter, (6) get the data, (7) save them to a .csv file, and (8) finalize the counter.

\section{Results}

We study performance and power efficiency on GPUs from different perspectives, studying the influence of a wide variety of issues, namely:

Table 5 Power, execution times and energy consumption on four GTX980 GPUs for different comparisons

\begin{tabular}{|c|c|c|c|c|c|}
\hline Comparison & Stage 1 & Stage 2 & Stage 3 & Total & \\
\hline & Average power (watts per GPU) & & & & GCUPS/W \\
\hline $\operatorname{chr} 22$ & $101.11 \mathrm{~W}$ & $116.26 \mathrm{~W}$ & $77.27 \mathrm{~W}$ & 101.33 & 0.55 \\
\hline $\operatorname{chr} 21$ & $102.11 \mathrm{~W}$ & $116.47 \mathrm{~W}$ & $78.89 \mathrm{~W}$ & 102.18 & 0.56 \\
\hline $47 \mathrm{M}$ & $104.37 \mathrm{~W}$ & $117.12 \mathrm{~W}$ & $76.33 \mathrm{~W}$ & 104.50 & 0.54 \\
\hline \multirow[t]{2}{*}{$\operatorname{chrY}$} & $103.25 \mathrm{~W}$ & $119.63 \mathrm{~W}$ & $77.00 \mathrm{~W}$ & 103.26 & 0.56 \\
\hline & Execution time (seconds) & & & & GCUPS \\
\hline $\operatorname{chr} 22$ & $11161.92 \mathrm{~s}$ & $185.20 \mathrm{~s}$ & $14.25 \mathrm{~s}$ & $11361.38 \mathrm{~s}$ & 224.60 \\
\hline $\operatorname{chr} 21$ & $9687.36 \mathrm{~s}$ & $61.49 \mathrm{~s}$ & $11.03 \mathrm{~s}$ & $9759.89 \mathrm{~s}$ & 229.25 \\
\hline $47 \mathrm{M}$ & $6694.95 \mathrm{~s}$ & $88.25 \mathrm{~s}$ & $9.05 \mathrm{~s}$ & $6792.26 \mathrm{~s}$ & 226.68 \\
\hline \multirow[t]{2}{*}{$\operatorname{chr} r$} & $6798.12 \mathrm{~s}$ & $3.99 \mathrm{~s}$ & $0.07 \mathrm{~s}$ & $6802.18 \mathrm{~s}$ & 229.93 \\
\hline & Energy consumption (kilojoules per GPU) & & & & Cost \\
\hline $\operatorname{chr} 22$ & $1128.63 \mathrm{~kJ}$ & $21.53 \mathrm{~kJ}$ & $1.10 \mathrm{~kJ}$ & $4 \times 1151.27 \mathrm{~kJ}$ & $0.1660 €$ \\
\hline $\operatorname{chr} 21$ & $989.26 \mathrm{~kJ}$ & $7.16 \mathrm{~kJ}$ & $0.87 \mathrm{~kJ}$ & $4 \times 997.29 \mathrm{~kJ}$ & $0.1440 €$ \\
\hline $47 \mathrm{M}$ & $698.82 \mathrm{~kJ}$ & $10.34 \mathrm{~kJ}$ & $0.69 \mathrm{~kJ}$ & $4 \times 709.85$ kJ & $0.1024 €$ \\
\hline $\operatorname{chry}$ & $701.94 \mathrm{~kJ}$ & $0.48 \mathrm{~kJ}$ & $0.00 \mathrm{~kJ}$ & $4 \times 702.42 \mathrm{~kJ}$ & $0.1012 €$ \\
\hline
\end{tabular}

We also use GCUPS (GigaCells Updated Per Second) as performance metric, GCUPS/W as power efficiency metric and overall costs in euros (shown for all GPUs involved and on an average fare of $0.13 € / \mathrm{kWh}$ ) 
Table 6 Power, execution times and energy consumption on different number of GTX980 GPUs for the chr22 comparison

\begin{tabular}{|c|c|c|c|c|c|c|}
\hline No. GPUs & Stage 1 & Stage 2 & Stage 3 & Total & Versus 2 GPUs & \\
\hline & Average power (watts) & & & & & GCUPS/W \\
\hline 4 & $101.11 \mathrm{~W}$ & $116.26 \mathrm{~W}$ & $77.27 \mathrm{~W}$ & $101.33 \mathrm{~W}$ & & 0.55 \\
\hline 3 & $101.53 \mathrm{~W}$ & $108.16 \mathrm{~W}$ & $78.79 \mathrm{~W}$ & $101.62 \mathrm{~W}$ & & 0.59 \\
\hline \multirow[t]{2}{*}{2} & $100.30 \mathrm{~W}$ & $114.68 \mathrm{~W}$ & $76.74 \mathrm{~W}$ & $100.38 \mathrm{~W}$ & & 0.57 \\
\hline & Execution time (seconds) & & & & & GCUPS \\
\hline 4 & $11161.92 \mathrm{~s}$ & $185.20 \mathrm{~s}$ & $14.25 \mathrm{~s}$ & $11361.38 \mathrm{~s}$ & $1.96 x$ & 224.59 \\
\hline 3 & $14719.32 \mathrm{~s}$ & $253.72 \mathrm{~s}$ & $17.70 \mathrm{~s}$ & $14990.76 \mathrm{~s}$ & $1.49 x$ & 170.21 \\
\hline \multirow[t]{2}{*}{2} & $22080.04 \mathrm{~s}$ & $159.77 \mathrm{~s}$ & $23.17 \mathrm{~s}$ & $22262.99 \mathrm{~s}$ & & 114.62 \\
\hline & Energy consumption (kilojoules) & & & & & Cost \\
\hline 4 & $1128.63 \mathrm{~kJ}$ & $21.53 \mathrm{~kJ}$ & $1.10 \mathrm{~kJ}$ & $4 \times 1151.27 \mathrm{~kJ}$ & $1.03 x$ & $0.1660 €$ \\
\hline 3 & $1494.60 \mathrm{~kJ}$ & $27.45 \mathrm{~kJ}$ & $1.40 \mathrm{~kJ}$ & $3 \times 1523.44 \mathrm{~kJ}$ & $1.02 x$ & $0.1650 €$ \\
\hline 2 & $2214.77 \mathrm{~kJ}$ & $18.32 \mathrm{~kJ}$ & $1.78 \mathrm{~kJ}$ & $2 \times 2234.88 \mathrm{~kJ}$ & & $0.1614 €$ \\
\hline
\end{tabular}

We also compare performance and power consumption versus the execution on 2 GPUs

1 The input data volume.

2 The computational stage within SW.

3 The multi-GPU factor.

4 The cost of speculative executions.

5 Comparison among GPUs (different generations and models).

6 Comparison with other accelerators (Xeon Phis) and low-power devices (FPGAs).

Now we dedicate a different subsection to analyze each of them separately.

\section{Influence of the input data set}

We have executed the modified CUDAlign 4.0 version using four different comparisons (see Table 4) on a multi-GPU environment composed of four GeForce GTX 980 GPUs. Table 5 includes the numbers coming from this first experiment. Wattage is slightly sensitive to workload, around 3\% higher on smaller comparisons (47M and chrY). Execution time is proportional to the number of Petacells computed on each comparison, and finally, energy consumption and costs follow this tendency too. Performance keeps stable around 225-230 GCUPS (Giga-Cell Updates per Second), and power efficiency remains constant in 0.55 GCUPS/W, which is not a great value, but the use of four GPUs here penalizes it. We will see higher efficiency on a single GPU later on.

Overall, we expect the compared sequences to play a more decisive role on smaller data volumes, say Mega-Cells or even Giga-Cells. But when exceeding the Peta-Cells threshold, the GPU already reaches a stationary behaviour that stabilizes power over time regardless of input/output transitions, and it is logical to find solid performance and power efficiency. Since the influence of the

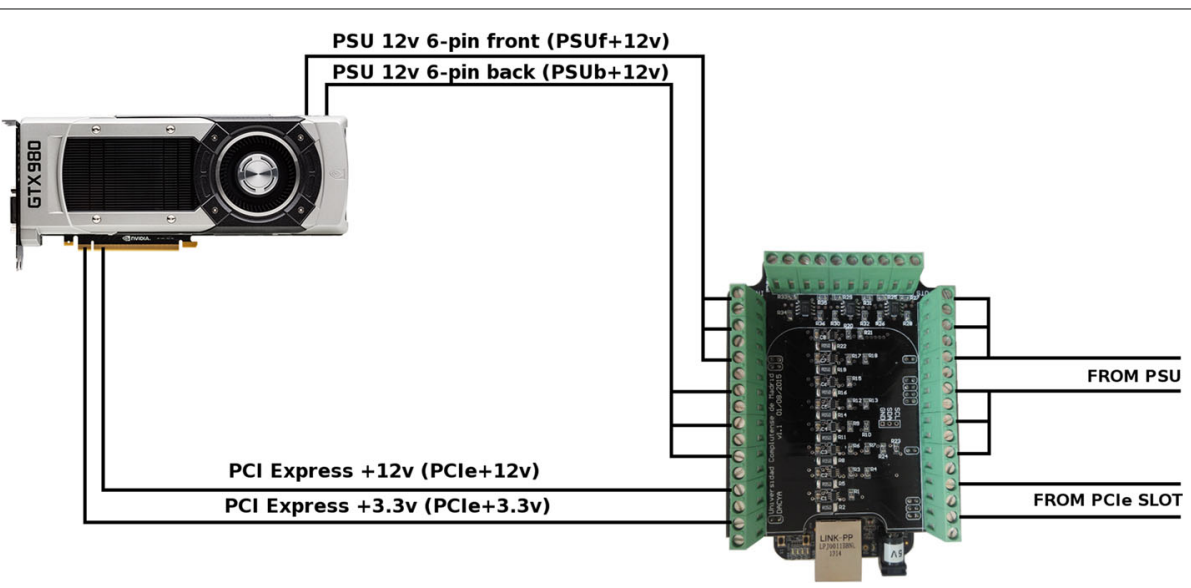

Fig. 6 Infrastructure for measuring energy on GPUs. Wires, slots, cables and connectors for measuring energy on GPUs 


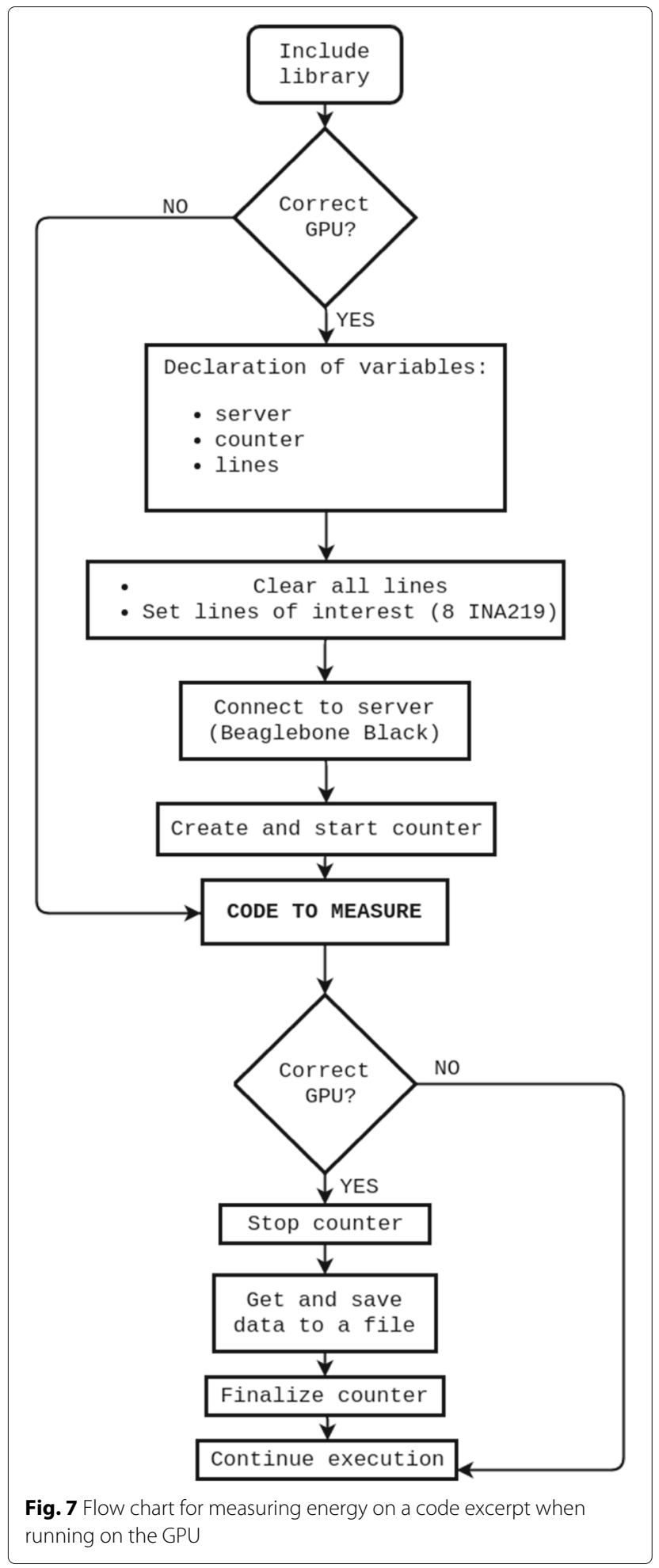

compared sequences is negligible, we will continue our analysis just using chr22 in remaining executions.

\section{Behaviour of every computational stage}

Table 5 also shows that stage 1 predominates for the execution time of SW, and that wattage keeps stable around 100 watts in that stage for all sequences. From that point, power goes up around $15 \%$ for stage 2 , and goes down around $25 \%$ for stage 3 . We find an explanation for this if we look at the energy budget for Kepler and Maxwell GPUs (see Table 7): Fetching operands costs more than computing on them. Therefore, stage 1 , which is the most computationally intensive, keeps on an intermediate point. In stage 2, where communications are more often, average power increases. And finally, we have stage 3, almost negligible in elapsed time, but performing selective operations with texture memory and disk as we already mentioned at the end of "Experimental setup" section. File operations are offloaded to a different subsystem of the computer, and they escape to our measurement system, so the GPU is mostly idle during that time, which reduces average power and compensates those expensive DRAM operations through a much larger time window.

Table 8 summarizes gains (in time reduction) and losses (as extra energy costs) on all scenarios of our multi-GPU execution for the chr22 sequence comparison, comparing 3 and 4 GPUs taking as baseline the first multi-GPU execution (2 GPUs). Stage 1 presented scalable results with a time reduction of almost $50 \%$ when doubling the number of GPUs from 2 to 4 , at the expense of less than $2 \%$ in the overall energy budget. Stage 2 slowdowns the execution time due the insufficient level of parallelism when using few GPUs, what causes a serial execution of the Stage 2 among GPUs. Since Stage 3 executes in parallel on each GPU, speedups increase up to $38.5 \%$. Finally, we have a solid conclusion on four GPUs, with time being reduced

Table 7 Energy budget on a $28 \mathrm{~nm}$. manufacturing process chip (all Kepler and Maxwell GPUs)

\begin{tabular}{|c|c|c|}
\hline & $\begin{array}{l}\text { Computational task } \\
\text { performed on the GPU }\end{array}$ & $\begin{array}{l}\text { Power consumption } \\
\text { (energy in picojoules) }\end{array}$ \\
\hline \multirow[t]{3}{*}{ Computation } & $\begin{array}{l}\text { Add operator using } \\
\text { integer operands (ALU) }\end{array}$ & 0.4 \\
\hline & $\begin{array}{l}\text { Mul operator using } \\
\text { fp64 operands (FPU) }\end{array}$ & 25 \\
\hline & $\begin{array}{l}\text { Fused multiply-add } \\
\text { on fp64 operands (FPU) }\end{array}$ & 40 \\
\hline \multirow[t]{3}{*}{ Data movement } & $\begin{array}{l}\text { Transition (milimeter } \\
\text { traversed per bit) }\end{array}$ & 0.2 \\
\hline & $\begin{array}{l}\text { On-chip } \\
\text { fp64 communication }[1, \\
10,20 \mathrm{~mm} .]\end{array}$ & {$[3,64,250]$} \\
\hline & Efficient off-chip link & 500 \\
\hline \multirow[t]{3}{*}{ Memory access } & $\begin{array}{l}\text { Local access to a } \\
\text { register file }\end{array}$ & 2 \\
\hline & $\begin{array}{l}\text { 256-bit access to on-chip } \\
8 \text { KB. SRAM cache }\end{array}$ & 50 \\
\hline & $\begin{array}{l}\text { DRAM read/write (for an } \\
\text { entire cache line) }\end{array}$ & 16000 \\
\hline
\end{tabular}


Table 8 Savings (in execution time) and penalties (in energy cost) when accelerating SW for the chr22 comparison on 4 and 3 GPUs taking as baseline the twin GPUs execution

\begin{tabular}{lllllllll}
\hline & Stage 1 & & Stage 2 & & Stage 3 & & Total \\
\hline No. GPUs & $\begin{array}{l}\text { Savings } \\
\text { (time) }\end{array}$ & $\begin{array}{l}\text { Penalty } \\
\text { (energy) }\end{array}$ & $\begin{array}{l}\text { Savings } \\
\text { (time) }\end{array}$ & $\begin{array}{l}\text { Penalty } \\
\text { (energy) }\end{array}$ & $\begin{array}{l}\text { Savings } \\
\text { (time) }\end{array}$ & $\begin{array}{l}\text { Penalty } \\
\text { (energy) }\end{array}$ & $\begin{array}{l}\text { Savings } \\
\text { (time) }\end{array}$ & $\begin{array}{l}\text { Penalty } \\
\text { (energy) }\end{array}$ \\
4 & $49.96 \%$ & $1.91 \%$ & $-15.92 \%$ & $135.04 \%$ & $38.50 \%$ & $23.60 \%$ & $48.97 \%$ & $3.03 \%$ \\
3 & $33.34 \%$ & $1.22 \%$ & $-58.80 \%$ & $124.75 \%$ & $23.61 \%$ & $17.98 \%$ & $32.67 \%$ & $2.25 \%$ \\
\hline
\end{tabular}

$50 \%$ at the expense of doubling the energy budget. Being Stage 1 the one with highest workload, the total execution time and energy usage follows its behaviour. Figure 8 provides details about the dynamic behaviour over time for each of the SW stages on different GPU models.

\section{Changing the number of GPUs}

Table 6 shows power and execution times when running SW for the chr22 comparison on a multi-GPU environment composed of 2, 3 and 4 GTX980 GPUs. We spend more than $6 \mathrm{~h}$ on two GPUs, and progressively reduce this time to less than a half using 4 GPUs.

As expected, power consumed by each GPU remains stable regardless of the number of GPUs active during the parallelization process. Execution times exhibit good scalability on stage 1 and 3 and somehow unstable for stage 2. Because GPUs keep computing on stage 1 most of the time, the overall energy cost is heavily influenced by this stage. Basically, doubling from 2 to 4 GPUs cuts execution times in half and doubles performance in GCUPS, with a small reduction on power efficiency: 0.57 GCUPS/W on two GPUs versus 0.55 GCUPS/W on four GPUs.

\section{Energy costs for speculative executions}

CUDAlign 4.0 executes the two SW algorithm phases ("Algorithm SW for local alignment" section) in six stages. In the first stage, the DP matrix is computed by multiple GPUs, which asynchronously communicate border elements to the right neighbour in order to find the optimal score. In the remaining stages, the traceback phase of SW speculates the location of the optimal alignment incrementally over the values calculated so far, thus anticipating results. Otherwise, its inherent serial execution would consume more than $50 \%$ of the overall execution time, depending on the sizes of the sequences. The speculative strategy, called IST (Incremental Speculative Traceback), has already been proven to be an effective mechanism for reducing the execution time, particularly on large-scale comparisons mapped to a wide number of GPUs [30].

To guarantee an effective reduction of the execution time, we speculate on two premises: (1) during the time the GPUs are otherwise idle, and (2) showing a very high speculation hit ratio. In terms of power consumption, the first premise consumes extra energy, which would only be amortized through a much shorter execution. And mispredictions may also jeopardize the GFLOPS/w ratio.

In order to analyze energy costs and establish amortization times for CUDAlign 4.0 in multi-GPU environments, we have monitored power consumption at real-time on a dual GPU execution performed on Titan Maxwell GPUs. Figure 9 shows the dynamic behaviour of a GPU when it speculates (IST - on the left) and when it does not (PT - on the right). We see that speculation skips inactivity (boxed on the right), and the GPU ends 18\% earlier. That produces average power to increase $11 \%$, but with energy savings of $6.5 \%$ when we speculate.

Table 9 summarizes pros and cons of the speculative approach. We need at least a $2 \mathrm{hit} / \mathrm{miss}$ ratio to waive energy penalties, and when doing so, we would save $12 \%$ of execution time as starting threshold.

\section{Comparison among GPUs}

Our next analysis compares different GPU generations and models. We have GPUs coming from three different generations (one Kepler, two Maxwells and one Pascal), and from two different budgets (two mid-end GTXs and two high-end Titans). Figure 8 illustrates the dynamic behaviour of power consumption for every GPU model (represented in rows, newer are lower) and CUDAlign stage (in columns).

Going stage by stage, we can see that:

1 Stage 1 reduces power in its final part on Titan GPUs.

2 Stage 2 has a different pattern on every GPU, predominating regions of lower power on newer GPUs, particularly at the end of the process.

3 Stage 3 shows the same pattern in all cases, and average power is higher on newer GPUs, but this is caused by a much higher throughput when computing and bandwidth when communicating, leading to power savings overall.

Table 10 provides more detailed results. In general, Titan Maxwell disappoints in power efficiency but fulfills 


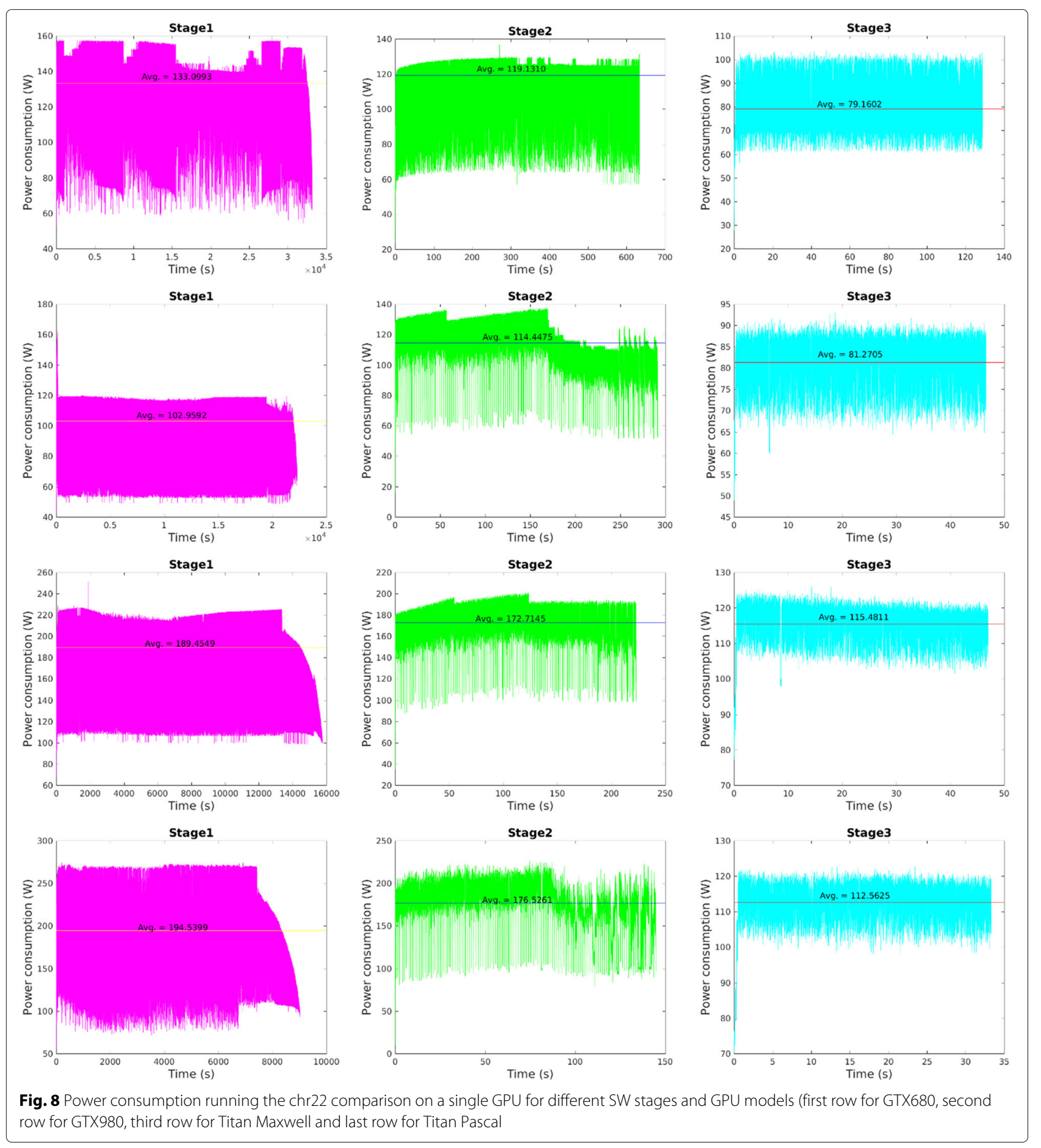

expectations in acceleration, whereas Titan Pascal is outstanding in both respects. We have built a GPU comparison taking GTX 980 (2015) as baseline (see sixth column in Table 10. Titan Maxwell (2016) improves 30\% the execution time but penalizes energy in the same percentage. Titan Pascal (2017) is able to reduce time by $59 \%$ and also energy by $23 \%$. Finally, Kepler, our
2013 model, increases execution time by $50 \%$ and almost doubles energy requirements.

Concerning power efficiency, GTX models behave better than Titans. And we are nicely surprised by energy costs: in less than five years, we have been able to reduce the energy cost of running our algorithm by as much as $60 \%$. 

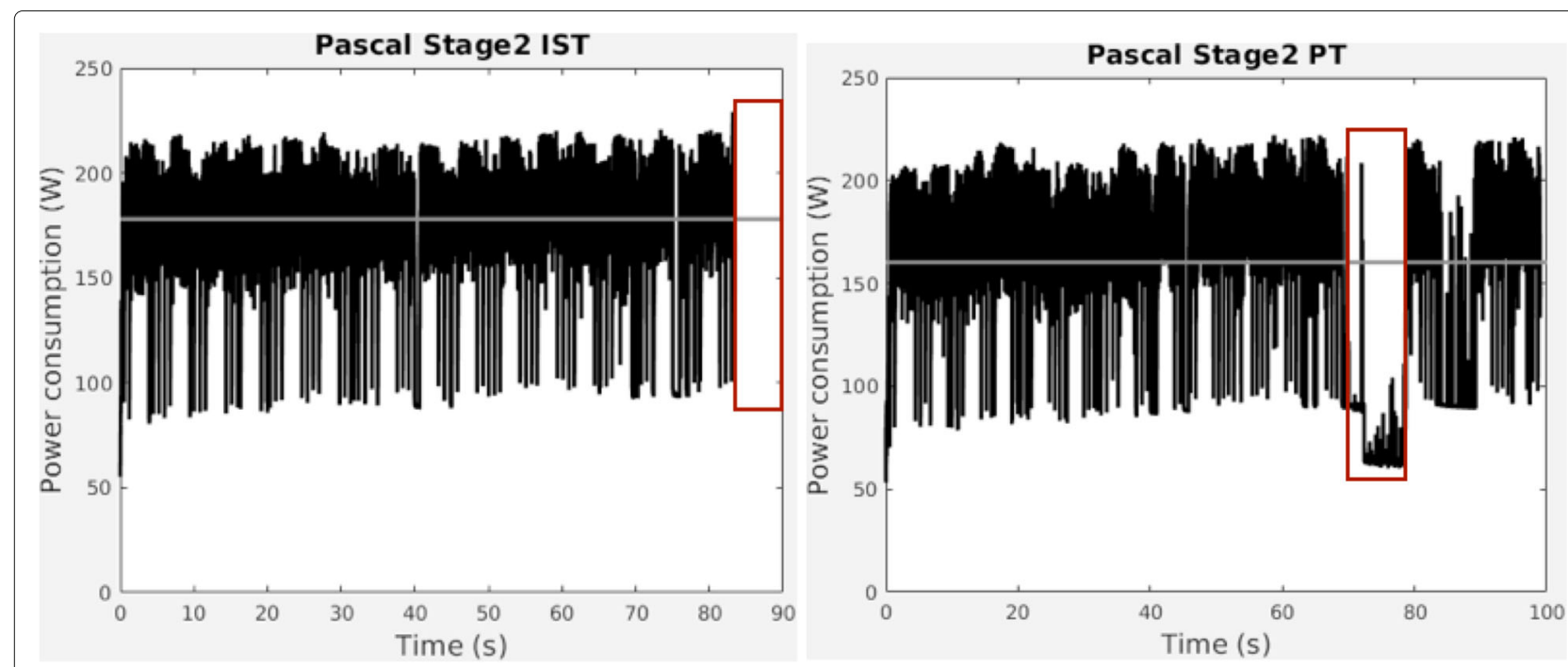

Fig. 9 Power consumption running the chr22 comparison on 2 Pascal GPUs without (right) and with (left) speculative execution

\section{Comparison with other devices and implementations}

For Megabase DNA sequences, the SW matrix is several Petabytes long, and so, few implementations allow alignments for DNA sequences longer than $10 \mathrm{MBP}$ (Million Base Pairs) like CUDAlign does.

SW\# [48] performed a CUDA implementation of dynamic programming algorithms for local alignment. With an emphasis on memory optimizations, SW\# was the first alternative to CUDAlign to produce sequence alignments on genome-wide scale, reporting a performance few hundred times faster than a counterpart CPU version.

More recently, Rucci et al. [34] have developed an OpenCL version of SW to study performance and power efficiency on Intel Xeon Phis and, overall, FPGAs, which has traditionally been the most power efficient devices for SW [27, 33]. Using an Altera Stratix V FPGA, they analyze the influence of data types for the matrix elements, showing that performance can be almost doubled when migrating from long int (32 bits) to int

Table 9 Speculative costs on a dual GPU execution using Pascal GPUs and chr22 comparison

\begin{tabular}{llll}
\hline Execution & Time & Avg. Power & Energy \\
Regular (PT) & $99.04 \mathrm{~s}$ & $160.21 \mathrm{~W}$ & $15867.19 \mathrm{~J}$ \\
Speculative (IST) & $83.39 \mathrm{~s}$ & $177.97 \mathrm{~W}$ & $14840.91 \mathrm{~J}$ \\
Comparison & $-18 \%$ & $+11 \%$ & $-6.5 \%$ \\
Spec. & Time & Avg. Power & Energy \\
Hit & $83.39 \mathrm{~s}$ & $177.97 \mathrm{~W}$ & $-6.5 \%$ \\
Miss & $99.04 \mathrm{~s}$ & $177.97 \mathrm{~W}$ & $+11.0 \%$ \\
\hline
\end{tabular}

(16 bits), and from here to char (8 bits). Unfortunately, those smaller data types overflow when using their scoring function on large sequences, so they conclude that FPGAs are faster than GPUs on small sequences (up to $200 \mathrm{~K}$ x $200 \mathrm{~K}$ matrices), and achieve the best GCUPS/W ratios.

Table 11 summarizes results for those counterpart implementations and also from previous CUDAlign versions. Running on the same device (the GTX 980 GPU), our code improves $1.39 \mathrm{x}$ performance and $2.25 \mathrm{x}$ power efficiency those results reported in [49]. And using the Pascal GPU, we are able to increase performance and power efficiency an additional $2.45 \mathrm{x}$ and $1.28 \mathrm{x}$ factors, respectively. Note that those results in Table 11 coming from other sources do not measure real power consumption like we do, but estimate it using the TDP reported by the manufacturer.

To dedicate few words to CPUs, Korpar et al. [48] already demonstrated that the CUDA version of SW running on GPUs is few hundred times faster than a counterpart CPU version, and given the fact that GPUs have conquered the green500.org list, we assume that CPUs are not competitive on power efficiency either. Even x86 many-cores, like the Xeon Phi 3120P endowed with 57 cores show the lowest performance and power efficiency of all configurations compared in Table 11.

FPGAs, on the other hand, are much slower than GPUs on large DNA sequences, but tough competitors regarding power efficiency. In fact, we compiled in "Related work" section several results where FPGAs clearly outperformed GPUs in GCUPS/W using GeForces coming from the first CUDA generation. Eight years and 
Table 10 Summary of CUDAlign stages, including the SW phase it belongs to and the processor where it is executed

\begin{tabular}{|c|c|c|c|c|c|c|}
\hline GPU model & Stage 1 & Stage 2 & Stage 3 & Total & Versus GTX 980 & \\
\hline & Average power (watts) & & & & & GCUPS/W \\
\hline GTX 680 (Kepler) & $133.10 \mathrm{~W}$ & $119.13 \mathrm{~W}$ & $79.16 \mathrm{~W}$ & $132.63 \mathrm{~W}$ & $+28 \%$ & 0.57 \\
\hline GTX 980 (Maxwell) & $102.95 \mathrm{~W}$ & $114.44 \mathrm{~W}$ & $81.27 \mathrm{~W}$ & $103.06 \mathrm{~W}$ & & 1.09 \\
\hline Titan X Maxwell & $189.45 \mathrm{~W}$ & $172.71 \mathrm{~W}$ & $115.48 \mathrm{~W}$ & $189.00 \mathrm{~W}$ & $+83 \%$ & 0.84 \\
\hline \multirow[t]{2}{*}{ Titan X Pascal } & $194.54 \mathrm{~W}$ & $176.52 \mathrm{~W}$ & $112.56 \mathrm{~W}$ & 193.96 W & $+88 \%$ & 1.42 \\
\hline & Execution time (seconds) & & & & & GCUPS \\
\hline GTX 680 (Kepler) & 33207.27 s & $642.37 \mathrm{~s}$ & $128.70 \mathrm{~s}$ & $33978.34 \mathrm{~s}$ & $+50 \%$ & 75.10 \\
\hline GTX 980 (Maxwell) & $22302.24 \mathrm{~s}$ & $291.50 \mathrm{~s}$ & $46.65 \mathrm{~s}$ & $22640.40 s$ & & 112.71 \\
\hline Titan X Maxwell & $15870.33 \mathrm{~s}$ & $222.11 \mathrm{~s}$ & $46.51 \mathrm{~s}$ & $16138.95 \mathrm{~s}$ & $-29 \%$ & 158.10 \\
\hline \multirow[t]{2}{*}{ Titan X Pascal } & $9114.21 \mathrm{~s}$ & $144.75 \mathrm{~s}$ & $33.02 \mathrm{~s}$ & $9291.98 \mathrm{~s}$ & $-59 \%$ & 274.62 \\
\hline & Energy consumption (kilojoules) & & & & & Cost \\
\hline GTX 680 (Kepler) & 4419.88 kJ & $76.52 \mathrm{~kJ}$ & 10.18 kJ & $4506.58 \mathrm{~kJ}$ & $+93 \%$ & $0.1627 €$ \\
\hline GTX 980 (Maxwell) & 2296.22 kJ & 33.36 kJ & $3.79 \mathrm{~kJ}$ & $2333.37 \mathrm{~kJ}$ & & $0.0842 €$ \\
\hline Titan X Maxwell & 3006.63 kJ & $38.36 \mathrm{~kJ}$ & $5.37 \mathrm{~kJ}$ & 3050.36 kJ & $+30 \%$ & $0.1101 €$ \\
\hline Titan X Pascal & $1773.07 \mathrm{~kJ}$ & $25.55 \mathrm{~kJ}$ & $3.71 \mathrm{~kJ}$ & $1802.33 \mathrm{~kJ}$ & $-23 \%$ & $0.0650 €$ \\
\hline
\end{tabular}

The comparison on sixth column takes GTX 980 as baseline

four generations later, GPU technology has turned around this situation. Table 12 summarizes speedups attained by GPUs and their deficit in energy versus FPGAs. We can see how performance gaps widens and power efficiency converges whenever GPUs reach contemporary models. And the programming effort on a FPGA is remarkable: 300 days versus just 45 days on a GPU for the implementation described in [27].

\section{Discussion and conclusions}

Along this paper, we have studied GPU acceleration and power consumption on a multi-GPU environment for the Smith-Waterman method to compute, via CUDAlign 4.0, the biological sequence alignment for a set of real genome scale DNA sequences coming from human and chimpanzee homologous chromosomes retrieved from the National Center for Biotechnology Information (NCBI).

We may distinguish 6 stages within CUDAlign 4.0, and the first half have been implemented in CUDA for its acceleration on GPUs. On a stage by stage analysis, the first one is more demanding and takes the bulk of the computational time. On the other hand, power consumption was kept more stable across executions of different alignment sequences, though it suffered deviations of up to $30 \%$ across different stages.

Table 11 Summary of SW implementations on accelerators and low-power devices over the past five years

\begin{tabular}{|c|c|c|c|c|c|c|c|}
\hline Device & Hardware model & Power & Implementation & Input size & GCUPS & GCUPS/W & Ref. \\
\hline FPGA & Altera Stratix V & $25 W^{(a)}$ & OpenCL & $23 \mathrm{M} \times 25 \mathrm{M}$ & 37.67 & 1.50 & {$[34]$} \\
\hline Accel. & Intel Xeon Phi 3120P & $270 W^{(a)}$ & OpenCL & $23 \mathrm{M} \times 25 \mathrm{M}$ & 30.36 & 0.12 & [34] \\
\hline GPU & Nvidia Tesla K20 & $225 W^{(a)}$ & SW\# & $23 \mathrm{M} \times 25 \mathrm{M}$ & 44.19 & 0.19 & [48] \\
\hline GPU & "Tesla K20 & $225 W^{(a)}$ & CUDAlign 3.0 & $23 \mathrm{M} \times 25 \mathrm{M}$ & 40.69 & 0.18 & [49] \\
\hline GPU & " GeForce GTX 980 & $165 W^{(a)}$ & SW\# & $23 \mathrm{M} \times 25 \mathrm{M}$ & 67.55 & 0.41 & [48] \\
\hline GPU & " GeForce GTX 980 & $165 W^{(a)}$ & CUDAlign 3.0 & $23 \mathrm{M} \times 25 \mathrm{M}$ & 84.84 & 0.51 & [49] \\
\hline GPU & " GeForce GTX 980 & $103.06 \mathrm{~W}$ & CUDAlign 4.0 & $51 \mathrm{M} \times 50 \mathrm{M}$ & 112.71 & 1.09 & \\
\hline GPU & "Titan X Maxwell & $189.00 \mathrm{~W}$ & CUDAlign 4.0 & $51 \mathrm{M} \times 50 \mathrm{M}$ & 158.10 & 0.84 & \\
\hline GPU & "Titan X Pascal & $193.96 \mathrm{~W}$ & CUDAlign 4.0 & $51 \mathrm{M} \times 50 \mathrm{M}$ & 276.53 & 1.43 & \\
\hline
\end{tabular}

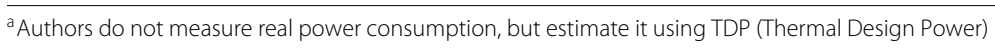


Table 12 GPUs contribution in acceleration and energy consumed versus a 2017 FPGA implementation using OpenCL

\begin{tabular}{llllll}
\hline Platform & FPGA & GTX 980 & GTX 980 & GTX 980 & Titan Pascal \\
Implementation & OpenCL & SW\# (CUDA) & CUDAlign 3.0 & CUDAlign 4.0 & CUDAlign 4.0 \\
Year & 2017 & 2013 & 2014 & 2016 & 2017 \\
Performance & Baseline & $+79.3 \%$ & $+125.2 \%$ & $+213.5 \%$ & $+669.2 \%$ \\
Power efficiency & Baseline & $-72.6 \%$ & $-66.0 \%$ & $-23.3 \%$ & $-1.3 \%$ \\
\hline
\end{tabular}

One of the major innovations in CUDAlign 4.0 was the Incremental Speculative Traceback (IST) [30] introduced within stage 2 to estimate the point where optimal alignment crosses border columns among multiple GPUs. This strategy allows GPUs to anticipate their activation, minimizing idle times required to solve dependencies when parallelizing. Mispredictions barely affect the execution time, but they may compromise power efficiency. On a dual GPU execution performed on Titan Maxwell GPUs, we find that a $2 \mathrm{hit} / \mathrm{miss}$ ratio is required to waive energy penalties, and in that case, we will also save $12 \%$ of the execution time.

In a multi-GPU environment composed of 4 GTX980 GPUs, we reduce the computational time by half when compared to a dual GPU execution, at the expense of just $3 \%$ penalty in power usage. That way, our experiments demonstrated an efficient correlation between acceleration and extra energy required.

Overall, we have reduced execution times from $9.5 \mathrm{~h}$ on a Kepler GPU to just $2.5 \mathrm{~h}$ on Titan Pascal, with energy costs cut by $60 \%$. Compared to FPGAs, which have an excellent reputation as low-power devices, GPUs are competitive and keep similar GFLOPS/w ratios in 2017 while maintaining the leadership as HPC accelerators for a five times faster execution.

We expect GPUs to increase their role as high performance and low power devices for biomedical applications in future GPU generations, particularly after the introduction in early 2017 of the 3D memory within Pascal models.

\section{Abbreviations \\ ASIC: Application specific integrated circuit; BLAST: Basic Local Alignment Search Tool; BLOSUM: Blocks Substitution Matrix; CUDA: Compute unified device arquitecture; DP: Dynamic programming; FPGA: Field programmable gate array; GCUPS: Giga-Cell Updates Per Second; GPGPU: General-purpose graphics processing unit; GPU: Graphics processing unit; HPC: High performance computing; IST: Incremental Speculative Traceback; MBP. Millions of Base Pairs; MM: Myers and Miller; NCBI: National Center for Biotechnology; NW: Needleman-Wunsch; PAM: Percent Accepted Mutations; PT: Pipeline Traceback; SW: Smith-Waterman}

\section{Acknowledgements}

We thank Nvidia for hardware donations within GPU Education Center 2011-2016 and GPU Research Center 2012-2016 awards at the University of Malaga (Spain). We thank Francisco D. Igual and Luis Piñuel from the Computer Architecture and Automated Department at the Complutense University of Madrid (Spain) for providing us Accelpower modules to measure power during our experimental survey. Our measuring system is based on a tool being continuously upgraded as reported in http://accelpowercape.dacya.ucm.es.

\section{Funding}

This work was supported by the Ministry of Education of Spain under Project TIN2013-42253-P and by the Junta de Andalucia under Project of Excellence P12-TIC-1741.

\section{Availability of data and materials}

The datasets used and/or analyzed during this study are available from the NCBI Website, http://www.ncbi.nlm.nih.gov. in particular, the Human Web Site cn be found at: https://www.ncbi.nlm.nih.gov/genome/gdv/?context= genome\&acc $=G C F \_000001405.36 \& c h r=22$ and the Chimpanze Website at: https://www.ncbi.nlm.nih.gov/genome/gdv/?context=genome\&acc=GCF_ $000001515.7 \& \mathrm{chr}=22$.

\section{About this supplement}

This article has been published as part of BMC Bioinformatics Volume 19 Supplement 14, 2018: Selected articles from the 5th International Work-Conference on Bioinformatics and Biomedical Engineering: bioinformatics. The full contents of the supplement are available online at https://bmcbioinformatics. biomedcentral.com/articles/supplements/volume-19-supplement-14.

\section{Authors' contributions}

Researchers of the University of Malaga performed the performance and energy measurements together with its experimental analysis. Researchers of the University of Brazilia performed the implementation of bioinformatics methods (CUDAlign) and discussed results. ACMAM and MU wrote most of the paper contents. All authors have read and approved the final manuscript.

\section{Ethics approval and consent to participate}

Not applicable.

\section{Consent for publication}

Not applicable (we have not taken material borrowed from other colleagues).

\section{Competing interests}

The authors declare that they have no competing interests.

\section{Publisher's Note}

Springer Nature remains neutral with regard to jurisdictional claims in published maps and institutional affiliations.

\section{Author details}

${ }^{1}$ Computer Architecture Department, University of Malaga, Malaga, Spain.

${ }^{2}$ Computer Science Department, University of Brasilia, Brasilia, Brazil.

Published: 20 November 2018

\section{References}

1. Deng X, Li J, Cheng J. Predicting Protein Model Quality from Sequence Alignments by Support Vector Machines. J Proteomics Bioinforma. 2013;9(2):.

2. Pierce B, Wiehe $K$, Hwang H, Kim B, Vreven T, Weng Z. ZDOCK Server: Interactive Docking Prediction of Protein-Protein Complexes and Symmetric Multimers. J Bioinforma. 2014;30(12):1771-3.

3. Li H, Homer N. A Survey of Sequence Alignment Algorithms for Next-generation Sequencing. Briefings in Bioinformatics. 2010;11(5): 473-83.

4. Wan P, Che D. Constructing Phylogenetic Trees Using Interacting Pathways. Bioinformation. 2013;9(7):363-7. 
5. Nvidia. CUDA Home Page. https://developer.nvidia.com/object/cuda. html. Accessed Apr 2018.

6. The Khronos Group. The OpenCL Core API Specification, Headers and Documentation. 2009. https://www.khronos.org/registry/cl. Accessed Apr 2018.

7. Pérez-Sánchez H, Wenzel W. Optimization methods for virtual screening on novel computational architectures. Curr Comput Aided Drug Des. 2011;7:44-52.

8. Merelli I, D’Agostino D, Pérez-Sánchez H, Gesing S. Managing, Analysing and Integrating Big Data in medical bioinformatics: open problems and future perspectives. Biomed Res Int.

9. Fang J, Varbanescu AL, Imbernón B, Cecilia JM, Pérez-Sánchez $\mathrm{H}$. Parallel Computation of Non-Bonded Interactions in Drug Discovery: Nvidia GPUs vs. Intel Xeon Phi. Granada: Proceedings of the $2^{\text {nd }}$ International Work-Conference on Bioinformatics and Biomedical Engineering (IWBBIO'14); 2014.

10. Benedict S. Energy-aware performance analysis methodologies for HPC architectures - An exploratory study. J Netw Comput Appl. 2012;35(6): 1709-19.

11. Pérez J, Sandes E, Melo A, Ujaldón M. Smith-Waterman Acceleration in Multi-GPUs: A Performance per Watt Analysis: Proceedings $5^{\text {th }}$ International Work-Conference on Bioinformatics and Biomedical Engineering (IWBBIO'17) Published as Lecture Notes in Bioinformatics, 10209, Part II, pp. 1-12.

12. Needleman S, Wunsch C. A General Method Applicable to the Search for Similarities in the Aminoacid Sequence of Two Proteins. J Mol Biol. 1970;48(3):443-453.

13. Smith T, Waterman M. Identification of Common Molecular Sequences. J Mol Biol. 1981;127(1):195-7.

14. NCBI. Blast: Basic local alignment search tool. 2017. https://blast.ncbi.nlm. nih.gov/Blast.cgi. Accessed Apr 2018.

15. Mount D. Bioinformatics: Sequence and Genome Analysis. Cold Spring Harbor Laboratory Press; 2004.

16. Gusfield D. Algorithms on Strings, Trees, and Sequences: Computer Science and Computational Biology. Cambridge University Press; 1997.

17. Durbin R, Eddy S, Krogh A, Mitchison G. Biological sequence analysis: Cambridge University Press; 1998.

18. Gotoh O. An Improved Algorithm for Matching Biological Sequences. J Mol Biol. 1982;162(3):705-8.

19. Hirschberg DS. A linear space algorithm for computing maximal common subsequences. Commun ACM. 1975;18(6):341-3.

20. Myers E, Miller W. Optimal Alignment in Linear Space. Comput Appl Biosci (CABIOS). 1988;4(1):11-17.

21. D Lipman D, Pearson W. Improved tools for biological sequence comparison. Proc Natl Acad Sci. 1988;85:2444-8.

22. Lipman D, Pearson W. Rapid and sensitive protein similarity searches. Science. 1985;227:1435-41.

23. Altschul SF, Gish W, Miller W, Myers EW, Lipman DJ. Basic local alignment search tool. J Mol Biol. 1990;215(3):403-10.

24. Altschul SF, et al. Gapped blast and psi-blast: A new generation of protein database search programs. Nucleic Acids Res. 1997;25(17):3389-402.

25. States DJ, Gish W. Combined use of sequence similarity and codon bias for coding region identification. J Comput Biol. 1997;1(1):39-50.

26. Hamidouche K, Machado F, Falcou J, Melo A, Etiemble D. Parallel Smith-Waterman Comparison on Multicore and Manycore Computing Platforms with BSP++. Intl. J Parallel Prog. 2013;41(1):111-36.

27. Benkrid K, Akoglu A, Ling C, Song Y, Liu Y, Tian X. High Performance Biological Pairsize Sequence Alignment: FPGA versus GPU versus Cell BE versus GPP. Intl. J Reconfigurable Comput. 201215. Article ID 752910 https://doi.org/10.1155/2012/752910.

28. Liu Y, Tam T, Lauenroth F, Schmidt B. SWAPHI-LS: Smith-Waterman Algorithm on Xeon Phi Coprocessors for Long DNA Sequences. IEEE Clust. 2014257-265

29. Sandes E, Melo A. Retrieving Smith-Waterman Alignments with Optimizations for Megabase Biological Sequences using GPU. IEEE Trans Parallel Distrib Syst. 2013;24(5):1009-21.

30. Sandes E, Miranda G, Martorell X, Ayguadé E, Teodoro G, Melo A. CUDAlign 4.0: Incremental Speculative Traceback for Exact Chromosome-Wide Alignment in GPU Clusters. IEEE Trans Parallel Distrib Syst. 2016;27(10):2838-50.
31. Cheah R, Halim A, Al-Junid S, Khairudin N. Design and Analysis of Low Powered DNA Sequence Alignment Accelerator Using ASIC. In: Proceedings 9th WSEAS Intl. Conference on Microelectronics, Nanoelectronics and Optoelectronics (MINO'10); 2010. p. 107-13.

32. Hasan L, Zafar H. Performance Versus Power Analysis for Bioinformatics Sequence Alignment. J Appl Res Technol. 2012;10(6):920-8.

33. Zou D, Dou Y, Xia F. Optimization Schemes and Performance Evaluation of Smith-Waterman Algorithm on CPU, GPU and FPGA. Concurr Comput Pract Experience. 2012;24:1625-44.

34. Rucci E, Botella G, de Giusti A, García C, Naiouf M, Prieto-Matías M. Accelerating Smith-Waterman Alignment of Long DNA Sequences with OpenCL on FPGA, vol. 2: Proceedings $4^{t} \boldsymbol{h}$ Intl. Work-Conference on Bioinformatics and Biomedical Engineering (IWBBIO'17); 2017, pp. 500-511.

35. Pfister G. In Search of Clusters: The Coming Battle in Lowly Parallel Computing: Prentice Hall; 1995.

36. NCBI. NCBI Web Site. 2017. https://www.ncbi.nlm.nih.gov. Accessed Apr 2018.

37. Prufer K, Munch K, et al. The bonobo genome compared with the chimpanzee and human genomes. Nature. 2012;486:527-31.

38. Nuttle X, Giannuzzi G, et al. Emergence of a Homo sapiens-specific gene family and chromosome 16p11.2 CNV susceptibility. Nature. 2016;536: 205-9.

39. Hirata S, Hirai H, Nogami E, Norimura N, Udono T. Chimpanzee Down syndrome: a case study of trisomy 22 in a captive chimpanzee. Primates. 2017;58(2):267-73.

40. Hallast $P$, Jobling A. The $Y$ chromosomes of the great apes. Hum Genet. 2017;136(5):511-28.

41. NCBI. NCBI Human Website. https://www.ncbi.nlm.nih.gov/genome/ gdv/?context=genome\&acc=GCF_000001405.36\&chr=22. Accessed Apr 2018.

42. NCBI. NCBI Chimpanze Website. https://www.ncbi.n/m.nih.gov/genome/ $\mathrm{gdv} /$ ?context=genome\&acc=GCF_000001515.7\&chr=22. Accessed Apr 2018.

43. BeagleBone. Beaglebone black. https://beagleboard.org/BLACK. Accessed Apr 2018.

44. González-Rincón J. Sistema basado en open source hardware para la monitorización del consumo de un computador. 2015. Master Thesis Project. Universidad Complutense de Madrid.

45. Ada L. Adafruit INA219 Current Sensor Breakout. https://learn.adafruit. com/adafruit-ina219-current-sensor-breakout. Accessed Apr 2018.

46. Igual F, Jara L, Gómez J, Piñuel L, Prieto M. A Power Measurement Environment for PCle Accelerators. Comput Sci Res Dev. 2015;30(2): $115-24$.

47. Alonso P, Badía R, Labarta J, Barreda M, Dolz M, Mayo R, Quintana-Ortí E, Reyes R. Tools for power-energy modelling and analysis of parallel scientific applications. In: Proceedings 41 st Intl. Conference on Parallel Processing (ICPP'12). IEEE Computer Society; 2012. p. 420-9.

48. Korpar M, Sikic M. SW\#-GPU-enabled exact alignmens on genome scale. J Bioinforma. 2013;29(19):2494-5.

49. Sandes E, Miranda G, Melo A, Martorell X, Ayguadé E. CuDAlign 3.0: Parallel Biological Sequence Comparison in Large GPU Clusters. In: Proceedings IEEE/ACM CCGrid'14; 2014. p. 160-9.

50. Sandes E, Melo A. CUDAlign: Using GPU to Accelerate the Comparison of Megabase Genomic Sequences. In: Proceedings 15th ACM SIGPLAN Symposium on Principles and Practice of Parallel Programming (PPoPP'10); 2010. p. 137-46.

51. Sandes E, Melo A. Smith-Waterman Alignment of Huge Sequences with GPU in Linear Space. In: Proceedings IEEE Intl. Parallel and Distributed Processing Symposium (IPDPS'11); 2011. p. 1199-211.

52. Sandes E, Miranda G, Martorell X, Ayguadé E, Teodoro G, Melo A. MASA: A Multi-Platform Architecture for Sequence Aligners with Block Pruning. ACM Trans Parallel Comput. 2016;2(4):28:1-28:31. 